\title{
RNA Interference: Antiviral Defense Mechanism and Immune Memory
}

\author{
Muratkhodjaev Javdat ${ }^{1,2, ~ *, ~ A r i p o v a ~ T a m a r a ~}{ }^{1}$ \\ ${ }^{1}$ Cellular Therapy Department, Institute of Immunology and Human Genomics, Tashkent, Uzbekistan \\ ${ }^{2}$ Research and Development Department of Pharmaceutical Company GENEX, Tashkent, Uzbekistan
}

Email address:

javdat_m@yahoo.com (M. Javdat)

${ }^{*}$ Corresponding author

To cite this article:

Muratkhodjaev Javdat, Aripova Tamara. RNA Interference: Antiviral Defense Mechanism and Immune Memory. Advances in Applied Physiology. Vol. 5, No. 2, 2020, pp. 24-29. doi: 10.11648/j.aap.20200502.13

Received: July 14, 2017; Accepted: August 5, 2017; Published: September 7, 2020

\begin{abstract}
A review of the mechanisms of the generation of antiviral immunity in bacteria, plants, invertebrates, and vertebrates directly indicates the leading role of innate immunity. Bornovirus infections in mammals have been proven to be inhibited by the RNA interference mechanism. Authors propose a possible role of innate human immunity in combating viral infections, including SARS-Cov2. This hypothesis is based on the notion that antiviral response involves a molecular mechanism of RNA interference stemming from the specific viral patterns incorporated into host cells' DNA. Innate immunity plays an important role not only at the first encounter with a viral infection, but also fully participates in the formation of specific immune memory. RNA-interference along with interferon system is integral parts of human anti-viral defense system. The joint work of these systems is considered. Caution is warranted in both PCR testing interpretation and in assessing prospective vaccines. False positive result without any clinical presentation of the disease in some people might mean that PCR test was picking up any specific SARS-Cov2 sequences that already had incorporated into asymptomatic person's cellular DNA due to the possible RNA-interference based anti-viral immunity. Future vaccines aimed at producing specific antibodies can cause the phenomenon of antibody-dependent enhancement (ADE) of the infection. In ADE virus not only infects susceptible cells through appropriate receptor, but is able to highjack virus-specific antibodies to easily traffic virus bodies inside the monocytes/macrophages, granulocytes, platelets, mast and many more host cells through interaction with $\mathrm{Fc}$ and/or complement receptors. It is necessary to draw the attention of medical community, especially practitioners to the role of innate immunity, which is especially important in the current COVID-19 pandemic.
\end{abstract}

Keywords: RNA-Interference, Innate Immune Memory, Antiviral Immunity, COVID-19, ADE

\section{Introduction}

\section{1. "Memory" of Innate Immunity}

The ability of innate immunity to remember and learn has been observed and intensively researched across various biological species. While plants and invertebrate animals do not possess features of adapted immunity, they are capable of forming systemic acquired resistance (SAR). In the studies by Chester in 1933 and by Ross in 1961 the primary infectious agent (IA) was shown to induce the SAR phenomenon upon exposure to the same or similar infectious agents $[6,7]$.

Invertebrates also response to the various IAs by modulating innate defense capacities [2-5]. Such strong alertness of immune system in invertebrates can last lifelong and further passes vertically and is detectable in offspring up to the third generation [9].

Finally, Mackaness 1964 has demonstrated an innate component contribution to the formation of immune memory in vertebrates [10]. Repeated administration of pathogenic microorganisms resulted in recruitment of highly sensitive macrophages, monocytes and NK-cells. Moreover, epigenetic changes in the effector cells themselves were noted [11, 12].

A complete overview of the innate memory is beyond the scope of this discussion, but several excellent reviews are available $[1,8,12]$. 


\subsection{Antiviral Defense Mechanisms}

Reviewing the antiviral defense mechanisms, it is necessary to single out the kingdom of bacteria that use the effective CRISPR-Cas system in the fight against viruses [13].

The CRISPR-Cas are adaptive immune systems with memory of past encounters with foreign DNA that is stored in unique spacer sequences derived from viral and plasmid genomes and inserted into CRISPR arrays. Transcripts of the spacers, along with portions of the surrounding repeats, are utilized as guide CRISPR (cr) RNAs to recognize the cognate sequences in foreign genomes and thus direct Cas nucleases to their unique cleavage sites [14].

A close analogue of such a protective system in eukaryotes might be a RNA interference [11]. Plants and invertebrates make extensive use of this mechanism against viruses [16-19].

Vertebrates, on the other hand, in addition to the antiviral RNA-i system [20-23] employ an interferon-activated antiviral system [24, 33, 34]. This protein-based defense system effectively combats viral infections, but it also blocks the operation of the anti-virus RNA-I system [24, 25, 35-38].

An analysis of published data allows us to hypothesize how specific antiviral memory might be generated via mechanism of RNA-interference

\section{Hypothesis of Specific Antiviral Memory Generation by Innate Immune System}

In our opinion, a key role in the fight against viral invasion is played by innate immunity, including physical barriers (tight joints in the skin, surfaces of the epithelium and mucous membranes, the mucus itself); anatomical barriers; enzymes of epithelial and phagocytic cells (e.g. lysozyme), phagocytes (neutrophils, monocytes, macrophages), serum proteins associated with inflammation (complement, C-reactive protein, lectins); antimicrobial peptides of surface and phagocytic granules (for example, defensins, etc.); cells that release cytokines and inflammatory mediators (i.e. macrophages, mast cells, NK cells) and cell receptors that sense the characteristic patterns of microorganisms (TLR, NLR, RLR, CLR receptors).

Among the latter, there are at least four TLR receptors that immediately respond to various components of viruses - TLR3 binds double-stranded (dsRNAs), TLR7 and TLR8 sense single-stranded (ssRNA), while TLR9 recognizes unmethylated CpG DNA. Furthermore, RLR receptors are intracellular proteins capable of perceiving viral dsRNA during virus replication. Each of these receptors ultimately activates the production of interferons, which trigger a cascade of defense reactions culminating in a cell apoptosis [29, 30]. When neighboring cells receive an interferon signal, they switch to the "alarm mode", which triggers an emergency halt in cellular protein synthesis, including the synthesis of viral proteins thus protecting a host cell from virus replication [40].
In addition to this interferon-based mechanism, an antiviral defense based on RNA interference might concurrently be working in the cell.

Exogenous viral RNAs are cleaved in the cytoplasm by the Dicer enzyme into short fragments of about 20 bases in length, called small interfering RNAs (siRNAs) [26].

These interfering RNAs bind with RNA-induced silencing complex (RISC). Active RISC ensures binding between interference RNA and a complementary viral sequence and consequently potentiates cutting a viral RNA by aid of Argonaute protein, ultimately inhibiting translation and/or RNA deadenyaltion [31, 32].

This mechanism has been proven to work with bornaviruses. In this case, the existing bornavirus sequences in the DNA of the target cells prevented the infection caused by bornavirus [41-44].

Furthermore, Parrish et al. raise the hypothesis that retrotransposon-dependent virus-to-host gene flow could engender RNA-mediated, sequence-specific antiviral immune memory in metazoans analogous to the CRISPR/Cas system in prokaryotes [43].

We assume that such interference-based cellular protection does not need millions of years to develop [42]. Quite an opposite, it forms rather quickly and locally, in response to current viral infection.

To do this, viral fragments must be reverse-transcribed and then inserted into host-cell DNA. The very existence of such mechanisms has been described in the research of retrotransposons and pseudogenes [27, 28], where intracellular reverse transcriptase ORF2 has been implemented in transforming cytoplasmic RNA and retroelement transcripts into complementary DNA parts.

Moreover, those retro-elements account to almost half of human DNA in the cells [29, 30].

It is tempting to assume then that at least some parts of human DNA are coded DNA fragments of previously encountered virus genome. This is some sort of "block list" of nucleotide sequences, prohibited for cell translation.

We propose to look for these sequences in the piRNA clusters. As shown by Gebert D and Rosenkranz D., there are many such piRNA clusters on chromosome 6 , which in a particular gene-dense region carries one of the largest and most strongly expressed piRNA clusters $[45,46]$

After transcription of these sequences mRNA is then cleaved by the Drosha nuclease into micro RNA (miRNAs) sized by 21-23 length fragments. Micro RNAs has been frequently observed during infections caused by both DNA and RNA viruses [20].

These interfering RNAs formed by Drosha (miRNA), would then bind with the same RISC complex. And, in the case of a current infection, the prepared RISK complex (when finding a match with the sequences of interfering RNAs), begins to rapidly cleave the viral RNA and inhibit the translation of viral proteins. This is a proposed mechanism of antiviral immune memory in a nutshell.

When delving into the aspect of viral localization in the body then at first seemingly contraindicated interferon and 
anti-viral RNA-I systems might work synergistically in anti-viral protection effort.

When virus enters the cell the mechanism of interferon release is initiated immediately triggering a myriad of cascade cellular responses culminating in a cell apoptosis [35, 36]. Neighboring cells also sense an interferon signal and in turn switch into an "alarm mode", which is usually block the very DICE/Drosha-RISC anti-viral protection system [37, 38].

However, as it turns out, this is not a case with actively proliferating and unipotent cells [39]. First line of cells that encounter virus entry is usually well differentiated surface epithelial or endothelial cells. They deal with the small virus load via interferon induced protection [33-38] and they do not participate in forming specific infection memory.

True immune memory is formed only in unipotent precursor cells when either virus enters in them or interference RNAs through extracellular vesicles [47].

Only de novo formed differentiated surface cells will possess specific antiviral memory giving them a powerful tool to effectively eliminate additional loads of virus. Maturation of the novel endothelial and epithelial cells usually takes several days which constitutes a required time for forming specific antiviral memory. It also means that this memory is local in its nature, and in order to have systemic memory multipotent cells should be involved.

Respectively, for innate immunity being able to protect further generations or off-spring, germ line cells should be impacted as well.

Therefore, in fact there is no any antagonism in the two antiviral protection systems of innate immunity. It all depends on the level of cell differentiation and location of these cells. Both methods of protection co-exist in the cells of the human body.

When the nucleotide sequences of the viral genes coincide with the retroelements present in the cell DNA, interference is triggered using the DICE / Drosha-RISC complex. This complex destroys the viral genome.

In the absence of a match, an interferon defense system is launched, which blocks the reproduction of the virus.

As noted above, with an initial high viral load, the infection is generalized, new retroelements corresponding to this virus are formed in progenitor cells using reverse transcriptase. When mature, these cells can use RNA-I in cases of repeated invasion of the virus.

\section{Discussion}

The above described mechanism of forming immune memory, by all means does not down value development of acquired immunity via specific CD4, CD8 cells along with humoral antibody-based antiviral immunity. This function of acquired immunity is well known, although the main role of this additional mechanism in vertebrates might lie with the preservation of the integrity of their own cells, control over their change during aging and destruction processes [48-50].

From this point, antibodies are produced against all antigens that a body encounters, including any viral proteins.
However, sometimes such "antiviral" antibodies are rather more harmful then protective. This is evident with the phenomenon of antibody-dependent enhancement (ADE) of the infection [51-54].

In $\mathrm{ADE}$ virus not only infects susceptible cells through appropriate receptor, but is able to highjack virus-specific antibodies to easily traffic virus bodies inside the monocytes/macrophages, granulocytes, platelets, mast and many more host cells through interaction with Fc and/or complement receptors [55]. There are numerous examples of ADE, triggered by alpha- and beta-coronaviruses [56, 57, 59].

Primates, vaccinated with modified Ankara vaccine virus encoding the full-length SARS-CoV glycoprotein, despite of low viral loads suffered from severe lung injury due to ADE [58]. ADE phenomenon has been observed on the animal models of SARS and MERS [52]. It has been shown that SARS-CoV-1 is capable to enter macrophages via antibody dependent route and even replicate in those cells $[57,58]$.

In connection with the foregoing, it is necessary to make adjustments to the assessment of herd immunity to COVID-19, which is at the moment traditionally based only on the measurement of theantibodies titers.

Another tool for this assessment should be DNA tests confirming the formation of new sequences in human cells corresponding to SARS-CoV-2 RNA. Future vaccines against SARS-CoV-2, the prospects of which are still remain elusive $[61,62]$, will also have to pass the same evaluation of effectiveness.

In our humble opinion, based on the proposed hypothesis above, the most effective anti-viral vaccines must be RNA vaccines since they would be able to induce RNA-interference and ultimately generating the strongest possible and hopefully long lasting immunity against the virus.

It also worth noting that if our hypothesis has any merits, then some of the observed false-positive results reported frequently during mass PCR diagnostics of Covid-19 infection might turn out to be just a laboratory artefact. False positive result without any clinical presentation of the disease in some people might mean that PCR test was picking up any specific SARS-Cov2 sequences that already had incorporated into asymptomatic person's cellular DNA due to the possible RNA-interference based anti-viral immunity. These people are, in fact, are non-contagious and safe for community.

\section{Conclusion}

This work forces us to abandon the dogma that has developed in medical immunology - only acquired immunity can form long-term memory. Numerous experimental data are presented that unequivocally indicate the leading role of innate immunity in the formation of specific memory. The RNA interference mechanism considered in detail is the main tool in the fight against viral infections and is responsible for the formation of long-term memory. This memory is based on specific cellular DNA patterns corresponding to the viral genome. It is tempting to assume then that at least some parts of human DNA are coded DNA fragments of previously 
encountered virus genome. This is some sort of "block list" of nucleotide sequences, prohibited for cell translation. These sequences can give false positive results in PCR testing of the current COVID-19 pandemic. In the case of a viral infection, acquired immunity, especially antibody production, is not the main defense. Moreover, specific antibodies can cause the phenomenon of antibody-dependent enhancement (ADE) of the infection. In ADE virus not only infects susceptible cells through appropriate receptor, but is able to highjack virus-specific antibodies to easily traffic virus bodies inside the monocytes/macrophages, granulocytes, platelets, mast and many more host cells through interaction with Fc and/or complement receptors. The conclusions for the medical community, especially medical practitioners, in the context of the COVID-19 pandemic may be as follows. Consider false positive PCR results and use convalescent serum and / or immunoglobulins with extreme caution to treat COVID-19.

\section{References}

[1] Reimer-Michalski EM, Conrath U. Innate immune memory in plants. Semin Immunol (2016) 28: 319-27. doi: 10.1016/j.smim.2016.05.006.

[2] Zhang T, Qiu L, Sun Z, Wang L, Zhou Z, Liu R, et al. The specifically enhanced cellular immune responses in Pacific oyster (Crassostreagigas) against secondary challenge with Vibrio splendidus. Dev Comp Immunol (2014) 45: 141-50. doi: 10.1016/j.dci.2014.02.015.

[3] Pope EC, Powell A, Roberts EC, Shields RJ, Wardle R, Rowley AF. Enhanced cellular immunity in shrimp (Litopenaeusvannamei) after "vaccination". PLoS One (2011) 6: e20960. doi: 10.1371/journal.pone.0020960.

[4] Wu G, Li M, Liu Y, Ding Y, Yi Y. The specificity of immune priming in silkworm, Bombyxmori, is mediated by the phagocytic ability of granular cells. J Insect Physiol (2015) 81: 60-8. doi: 10.1016/j.jinsphys.2015.07.004.

[5] Kurtz J. Specific memory within innate immune systems. Trends Immunol (2005) 26: 186-92. doi: 10.1016/j.it.2005.02.001.

[6] Chester KS. The problem of acquired physiological immunity in plants. Q Rev Biol. 1933; 8: 275-324.

[7] Ross AF. Localized acquired resistance to plant virus infection in hypersensitive hosts. Virology. 1961; 14: 329-339.

[8] Diana Boraschi and Paola Italiani. Innate Immune Memory: Time for Adopting a Correct Terminology. Front. Immunol., 19 April 2018 | https: //doi.org/10.3389/fimmu.2018.00799.

[9] Agrawal AA, Laforsch C, Tollrian R. Transgenerational induction of defences in animals and plants. Nature (1999) 401: 60-3. doi: $10.1038 / 43425$.

[10] Mackaness G. B. The immunological basis of acquired cellular resistance. J. Exp. Med. 1964; 120: 105-120.

[11] Cynthia Castro-Vargas et al. Potential Mechanism Related to Immune Priming within But Not across Generations. Front. Microbiol., 28 March 2017. doi.org/10.3389/fmicb.2017.00473.
[12] Mihai G. Netea, Jessica Quintin, Jos W. M. van der Meer. Trained Immunity: A Memory for Innate Host Defense. PERSPECTIVE| VOLUME 9, ISSUE 5, P355-361, MAY 19, 2011 doi.org/10.1016/j.chom.2011.04.006.

[13] RodolpheBarrangou. The Roles of CRISPR-Cas Systems in Adaptive Immunity and Beyond. Current Opinion in Immunology. 32: 36-41. doi: 10.1016/j.coi.2014.12.008.

[14] Eugene V. Koonin, Kira S. Makarova. Mobile Genetic Elements and Evolution of CRISPR-Cas Systems: All the Way There and Back. Genome Biology and Evolution, Volume 9, Issue 10, October 2017, Pages 2812-2825, doi.org/10.1093/gbe/evx192.

[15] Fire A., Xu S., Montgomery M., Kostas S., Driver S., Mello C. Potent and specific genetic interference by double-stranded RNA in Caenorhabditiselegans. Nature. 1998. Vol. 391, 6669. P. 806-811. doi: $10.1038 / 35888$.

[16] Byung-Chun Yoo et al. A Systemic Small RNA Signaling System in Plants. Plant Cell. 2004 Aug; 16 (8): 1979-2000. doi: 10.1105/tpc.104.023614.

[17] Shou-Wei Dingand Olivier Voinnet. Antiviral Immunity Directed by Small RNAs. Cell. 2007 Aug 10; 130 (3): 413-426. doi: $10.1016 /$ j.cell.2007.07.039.

[18] Cordula Kemp and Jean-Luc Imler. Antiviral immunity in drosophila. CurrOpinImmunol. 2009 Feb; 21 (1): 3-9. Published online 2009 Feb 14. doi: 10.1016/j.coi.2009.01.007.

[19] Peter Sarkies, Eric A Miska. RNAi Pathways in the Recognition of Foreign RNA: Antiviral Responses and Host-Parasite Interactions in Nematodes. BiochemSoc Trans. 2013 Aug; 41 (4): 876-80. doi: 10.1042/BST20130021.

[20] RoghiyhAliyari and Shou-Wei Ding. RNA-based viral immunity initiated by the Dicer family of host immune receptors. Immunol Rev. 2009 Jan; 227 (1): 176-188. doi: 10.1111/j.1600-065X.2008.00722.

[21] Pierre V Maillard, Annemarthe G van der Veen, Enzo Z Poirier, Caetano Reis e Sousa. Slicing and dicing viruses: antiviral RNA interference in mammals. EMBO J.2019. 38: e100941 doi.org/10.15252/embj.2018100941.

[22] Zhang Y, Li Y. Regulation of innate receptor pathways by microRNAs. Sci China Life Sci. 2013 Jan; 56 (1): 13-8. doi: 10.1007/s11427-012-4428-2. Epub 2012 Dec 27.

[23] Yingke Li, Xueyin Shi. Micro RNAs in the Regulation of TLR and RIG-I Pathways. Cell MolImmunol. 2013 Jan; 10 (1): 65-71. doi: 10.1038/cmi.2012.55.

[24] Jiaxi Wu, Zhijian J Chen. Innate Immune Sensing and Signaling of Cytosolic Nucleic Acids. Annu Rev Immunol. 2014; 32: 461-88. doi: 10.1146 /annurev-immunol-032713-120156.

[25] Annemarthe G van der Veen et al. The RIG-I-like receptor LGP2 inhibits Dicer-dependent processing of long double-stranded RNA and blocks RNA interference in mammalian cells. EMBO J. 2018 Feb 15; 37 (4): e97479. doi: 10.15252/embj.201797479.

[26] Ian G. Cannell, Yi Wen Kong, Martin Bushell. How do microRNAs regulate gene expression? Biochem Soc Trans (2008) $36 \quad$ (6): 1224-1231. https://doi.org/10.1042/BST0361224. 
[27] LalehHabibi and HamzehSalmani. Pivotal Impacts of Retrotransposon Based Invasive RNAs on Evolution. Front. $\begin{array}{llll}\text { Microbiol., } & 10 & \text { October } & 2017\end{array}$ doi.org/10.3389/fmicb.2017.01957.

[28] W Wei, T A Morrish, R S Alisch, J V Moran. A Transient Assay Reveals That Cultured Human Cells Can Accommodate Multiple LINE-1 Retrotransposition Events. Anal Biochem. 2000 Sep 10; 284 (2): 435-8. doi: 10.1006/abio.2000.4675.

[29] Lander ES, Linton LM, Birren B, Nusbaum C, Zody MC, Baldwin J, et al. (February 2001). "Initial sequencing and analysis of the human genome". Nature. 409 (6822): 860-921. doi: $10.1038 / 35057062$.

[30] Wicker T, Sabot F, Hua-Van A, Bennetzen JL, Capy P, Chalhoub B, Flavell A, Leroy P, Morgante M, Panaud O, Paux E, San Miguel P, Schulman AH (December 2007). "A unified classification system for eukaryotic transposable elements". Nature Reviews. Genetics. 8 (12): 973-82. doi: $10.1038 / \operatorname{nrg} 2165$.

[31] Song, Ji-Joon, Stephanie K. Smith, Gregory J. Hannon, Leemor Joshua-Tor. 2004. Crystal Structure of Argonaute and Its Implications for RISC Slicer Activity. Science 305: 1434-1437.

[32] Meister, Gunter, and Thomas Tuschi. 2004. Mechanisms of Gene Silencing by Double Stranded RNA. Nature 431: 343-349.

[33] K Onomoto, M Yoneyama, T Fujita. Regulation of Antiviral Innate Immune Responses by RIG-I Family of RNA Helicases. Curr Top MicrobiolImmunol. 2007; 316: 193-205. doi: 10.1007/978-3-540-71329-6_10.

[34] Agnieszka Jabłońska, Edyta Paradowska. Role of the RIG-I-like Receptors in Antiviral Response. Postepy Hig Med Dosw (Online). 2014 Jan 2; 68: 541-56. DOI: $10.5604 / 17322693.1102281$.

[35] Amir Apelbaum, GanitYarden, ShiraWarszawski, Daniel Harari, and Gideon Schreiber. Type I Interferons Induce Apoptosis by Balancing cFLIP and Caspase- 8 Independent of Death Ligands. Mol Cell Biol. 2013 Feb; 33 (4): 800-814. doi: 10.1128/MCB.01430-12.

[36] Kevin P. Kotredes and Ana M. Gamero. Interferons as Inducers of Apoptosis in Malignant Cells. J Interferon Cytokine Res. 2013 Apr; 33 (4): 162-170. doi: 10.1089/jir.2012.0110.

[37] David E. Levy. Whence Interferon? Variety in the Production of Interferon in Response to Viral Infection. J Exp Med. 2002 Feb 18; 195 (4): f15-f18. doi: 10.1084/jem.20020075.

[38] Daniel B. Stetson and RuslanMedzhitov. Type IInterferons in Host Defense. Immunity. Volume 25, Issue 3, September 2006, Pages 373-381. doi.org/10.1016/j.immuni.2006.08.007.

[39] Julie Eggenberger, Daniel Blanco-Melo, MarylinePanis, Kristen J. Brennand, and Benjamin R. tenOever. Type I interferon response impairs differentiation potential of pluripotent stem cells. PNAS January 22, 2019116 (4) 1384-1393; doi.org/10.1073/pnas.1812449116.

[40] RuslanMedzhitov. Approaching the Asymptote: 20 Years Later Immunity. 2009 Jun 19; 30 (6): 766-75. doi: 10.1016/j.immuni.2009.06.004.

[41] KanFujino, Masayuki Horie, Tomoyuki Honda, Dana K. Merriman, and KeizoTomonaga. Inhibition of Borna disease virus replication by an endogenous bornavirus-like element in the ground squirrel genome. PNAS September 9, 2014111 (36) 13175-1318, doi.org/10.1073/pnas.1407046111.

[42] Tomoyuki HondaandKeizoTomonaga. Endogenous Non-Retroviral RNA Virus Elements Evidence a Novel Type of Antiviral Immunity. Comment Mob Genet Elements. 2016 $\begin{array}{lllll}\text { Mar 22; } 6 & \text { (3): } & \text { e1165785. doi: }\end{array}$ 10.1080/2159256X.2016.1165785.

[43] NICHOLAS F. PARRISH et al. piRNAs derived from ancient viral processed pseudogenesas transgenerational sequence-specific immune memory in mammals. RNA. 2015. (10): 1691-703. doi: 10.1261/rna.052092.115.

[44] Horie M, Kobayashi Y, Suzuki Y, Tomonaga K. Comprehensive analysis of endogenous bornavirus-like elements in eukaryote genomes. Philos Trans R SocLond B Biol Sci. 2013; 368 (1626): 20120499. Published 2013 Aug 12. doi: $10.1098 /$ rstb.2012.0499.

[45] Daniel Gebert, Hans Zischler, David Rosenkranz. Primate piRNA Cluster Evolution Suggests Limited Relevance of Pseudogenes in piRNA-Mediated Gene Regulation. Genome Biology and Evolution, Volume 11, Issue 4, April 2019, Pages 1088-1104, https://doi.org/10.1093/gbe/evz060.

[46] Gebert D, Rosenkranz D. RNA-based regulation of transposon expression. Wiley Interdiscip Rev RNA. 2015; 6 (6): 687-708. doi: 10.1002/wrna.1310.

[47] Holtzman, J., Lee, H. Emerging role of extracellular vesicles in the respiratory system. Exp Mol Med (2020). https://doi.org/10.1038/s12276-020-0450-9.

[48] Irun R. Cohen and Douglas B. Youngb. Autoimmunity, microbial immunity and the immunological homunculus. Immunology Today. Volume 12, Issue 4, April 1991, Pages 105-110. doi.org/10.1016/0167-5699(91)90093-9.

[49] А. М. Зайчик, А. Б. Полетаев, Л. П. Чурилов. РАСПОЗНАВАНИЕ «СВОЕГО» И ВЗАИМОДЕЙСТВИЕ СО «СВОИМ» КАК ОСНОВНАЯ ФОРМА АКТИВНОСТИ АДАПТИВНОЙ ИММУННОЙ СИСТЕМЫ. (Self recognition and self interaction as the main form of immune system activity). ВестникСПбГУ. Сер. 11. 2013. Вып. 1 УДК 612.017.1+616.097+576.8.

[50] Галактионов В. Г. Проблемыэволюционнойбиологии. Медицинскаяиммунология. 2004, том 6, №3-5.

[51] HawkesR.

A. Enhancementoftheinfectivityofarbovirusesbyspecificantiserapr oducedindomesticfowls. Aust. J. Exp. Biol. Med. Sci. 42, 465482. DOI: $10.1038 /$ icb.1964.44.

[52] Maria K. Smatti, Asmaa A. Al Thani, and Hadi M. Yassine. Viral-Induced Enhanced Disease Illness. Front Microbiol. 2018; 9: 2991. doi: 10.3389/fmicb.2018.02991.

[53] Vennema Harry; Poland Amy; Foley Janet; Pedersen NielsC."Feline Infectious Peritonitis Viruses Arise by Mutation from Endemic Feline Enteric Coronaviruses". Virology. 1998. 243 (1): 150-157. doi: 10.1006/viro.1998.9045.

[54] Tirado S. M. andYoonK. J. "Antibody-dependent enhancement of virus infection and disease". Viral Immunology. 2003. 16 (1): 69-86. doi: 10.1089/088282403763635465. 
[55] Khandia R.; Munjal A.; Dhama K.; Karthik K.; Tiwari R.; Malik Y. S.; Singh R. K.; Chaicumpa W. "Modulation of Dengue/Zika Virus Pathogenicity by Antibody-Dependent Enhancement and Strategies to Protect Against Enhancement in Zika Virus Infection". Frontiers in Immunology. 2018. 9: 597. doi: 10.3389/fimmu.2018.00597.

[56] Iwasaki Akiko; Yang Yexin. "The potential danger of suboptimal antibody responses in COVID-19". Nature Reviews Immunology. 2020-04-21 doi: 10.1038/s41577-020-0321-6.

[57] Tseng C. et al. "Immunization with SARS Coronavirus Vaccines Leads to Pulmonary Immunopathology on Challenge with the SARS Virus". PLOS ONE. 2012.7 (4): e35421. Bibcode: 2012PLoSO...735421T doi: 10.1371/journal.pone.0035421.

[58] Liu L. et al. "Anti-spike IgG causes severe acute lung injury by skewing macrophage responses during acute SARS-CoV infection". JCI Insight. $2019.4 \quad$ (4). doi: 10.1172/jci.insight. 123158 .
[59] Wan Y. et al. "Molecular Mechanism for Antibody-Dependent Enhancement of Coronavirus Entry". Journal of Virology. 2020. 94 (5). doi: 10.1128/JVI.02015-19.

[60] YipMing S; Cheung Chung Y; Li Ping H; Bruzzone Roberto; Peiris JS Malik; Jaume Martial. "Investigation of Antibody-Dependent Enhancement (ADE) of SARS coronavirus infection and its role in pathogenesis of SARS". BMC Proceedings. 2011-01-10. 5 (S1). doi: 10.1186/1753-6561-5-s1-p80.

[61] Lynne Peeples. News Feature: Avoiding pitfalls in the pursuit of a COVID-19 vaccine. ProcNatlAcadSci U S A. 2020 Apr 14; 117 (15): 8218-8221. doi: 10.1073/pnas.2005456117.

[62] Peter J. Hotez, David B. Corry and Maria Elena Bottazzi. COVID-19 vaccine design: the Janus face of immune enhancement. Nat Rev Immunol. 2020 Apr 28: 1-2. doi: 10.1038/s41577-020-0323-4. 